Vol. 5, $n^{\circ} 1 \mid 2011$

TIC et genre

\title{
Cycles de vie et carrières dans les métiers des TIC : une perspective de genre
}

Gérard Valenduc

\section{CpenEdition}

Journals

Édition électronique

URL : http://journals.openedition.org/ticetsociete/939

DOI : 10.4000/ticetsociete.939

Éditeur

Association ARTIC

Référence électronique

Gérard Valenduc, «Cycles de vie et carrières dans les métiers des TIC : une perspective de genre »,

tic\&société [En ligne], Vol. 5, n 1 | 2011, mis en ligne le 27 mai 2019, consulté le 10 décembre 2020

URL : http://journals.openedition.org/ticetsociete/939; DOI : https://doi.org/10.4000/ticetsociete.939 


\title{
Cycles de vie et carrières dans les métiers des TIC : une perspective de genre
}

\author{
Gérard VALENDUC \\ Codirecteur du Centre de recherche de la Fondation Travail-Université (FTU, \\ Namur, Belgique) \\ Professeur invité à l'Université de Namur (FUNDP) et à l'Université de Louvain- \\ la-Neuve (UCL) \\ FTU, Rue de l'Arsenal, 5, B-5000 Namur \\ gvalenduc@ftu-namur.org
}

Gérard Valenduc est docteur en informatique. II s'est spécialisé dans la recherche sur la dimension humaine, sociale et politique des changements technologiques. II a participé à de nombreux projets concernant les aspects sociaux et sociétaux de l'innovation technologique, à l'échelle régionale, nationale ou européenne. Ses recherches et publications concernent les mutations du travail liées à la diffusion des technologies de l'information et de la communication, l'exclusion et l'inclusion numériques, les relations entre technologie et société. 


\title{
Cycles de vie et carrières dans les métiers des TIC : une perspective de genre
}

\begin{abstract}
Résumé
Malgré diverses campagnes de sensibilisation, les femmes sont de moins en moins nombreuses à la fois dans les métiers traditionnels de l'informatique, dans les nouveaux métiers de l'internet et de l'e-business, ainsi que dans les études qui mènent à ces métiers. La détérioration de la situation des femmes est analysée à la lumière des changements globaux qui affectent aujourd'hui les métiers des TIC. Trois tendances sont soulignées : l'élargissement du portefeuille de compétences, la diversification des modèles de carrière et l'évolution de la culture professionnelle. Elles ont des effets différenciés sur les femmes et les hommes. Elles modifient également les conditions d'entrée et de sortie des femmes dans les métiers des TIC. En conclusion, l'article plaide pour une approche en termes de cycle de vie, dans le double objectif d'améliorer les connaissances sur le genre dans les carrières des TIC et de rendre plus efficaces et plus ciblées les politiques en faveur de l'égalité professionnelle.
\end{abstract}

Mots clés : genre, métiers des TIC, carrières, inégalités professionnelles, politiques d'égalité

\begin{abstract}
Despite various awareness campaigns, women are increasingly underrepresented in traditional computer professions, new professions of the Internet and e-business, and education curricula leading to these jobs. The analysis of the worsening women's situation is highlighted by global changes affecting nowadays ICT professions. Three trends are underlined: the broadening of the skills portfolio, the diversification of career patterns, and the evolution of professional culture. They have differentiated effects on women and men. They modify the entry and exit paths in ICT professions. The conclusion emphasises a life cycle approach, aiming at the improvement of both knowledge about gender in ICT careers and targeted efficiency of job equality policies.
\end{abstract}


Cycles de vie et carrières dans les métiers des TIC : une perspective de genre

Keywords: gender, ICT professions, careers, gender gap, equality policies

\section{Resumen}

A pesar de varias campañas de sensibilización, mujeres son cada vez menos numerosas en ambas profesiones informáticas clásicas y profesiones nuevas del multimedia y e-business, tal como en los estudios que conducen a esos oficios. Las perspectivas para las mujeres son analizadas frente a los cambios globales que están afectando hoy las carreras de las TIC. Se destacan tres tendencias: la ampliación del abanico de competencias, la diversificación de modelos de carrera y la evolución de la cultura profesional. Tienen efectos diferenciados para mujeres y hombres. Modifican también las condiciones de entrada y salida de mujeres en las carreras de las TIC. La conclusión propone un enfoque en el concepto de ciclo de vida, para mejorar el conocimiento de la dimensión de genero en el desarrollo de las carreras profesionales en las TIC y para fomentar políticas más eficaces y precisas en el ámbito de la igualdad profesional.

Palabras claves : genero, profesiones de las TIC, carreras, desigualdades profesionales, políticas de igualdad. 
Pourquoi y a-t-il de moins en moins de femmes dans les métiers des technologies de l'information et de la communication (TIC), tandis qu'elles sont de plus en plus nombreuses dans les professions scientifiques et dans l'ingénierie ? Pourquoi les jeunes filles s'engagent-elles si rarement dans des études qui mènent aux métiers des TIC, alors qu'elles utilisent internet aussi assidument que les jeunes garçons ? Pourquoi les carrières des femmes dans ces métiers sont-elles caractérisées à la fois par le plafond de verre (obstacles peu visibles à la progression professionnelle) et le tuyau percé (abandons et changements d'orientation)? Ces questions ne sont pas neuves et c'est précisément leur récurrence et leur aggravation qui posent problème.

Après une introduction sur l'état actuel des recherches sur la place des femmes dans les métiers des TIC, cet article fera le point sur les paradoxes qui caractérisent la situation des femmes, puis passera en revue quelques grandes tendances dans l'évolution récente des métiers des TIC, qui ont des implications différenciées pour les hommes et les femmes, en termes de carrière et de développement professionnel. Enfin, il s'intéressera aux diverses formes d'entrée et de sortie des femmes dans ces métiers. En conclusion, il montrera l'intérêt d'une approche en termes de cycle de vie.

\section{Un bref état des recherches}

Au cours des dix dernières années, de nombreuses recherches ont été consacrées aux disparités de genre dans les métiers des TIC. Certains travaux se sont focalisés sur l'éducation et la formation, mettant en évidence les représentations sexuées de l'informatique et leurs impacts sur les orientations scolaires et académiques (Cohoon, 2009 ; Collet, 2006 ; Gras-Velazquez et al., 2009 ; Kirkup, 2010). D'autres recherches se sont centrées sur le monde du travail. Elles ont pris en considération les conditions d'emploi et de travail des femmes, les carrières, l'équilibre entre travail et vie privée. Elles ont ainsi mis en évidence les discriminations ou les inégalités professionnelles dont les femmes sont victimes dans les métiers des TIC (Castaño et al., 2009 ; Ponzellini et al., 2006 ; Valgaeren, 2007 ; Valenduc et al., 2004b ; Webster J., 2003). D'autres recherches encore ont intégré la question des métiers des TIC dans une approche plus large en termes d'inclusion ou d'exclusion numérique, s'intéressant aux inégalités entre les hommes et les femmes dans d'accès aux TIC et dans leurs usages (Castaño, 2008 ; Sørensen et Stewart, 2002). Enfin, certaines études de genre ont abordé le caractère sexué des concepts et des méthodes dans le domaine de l'informatique et du multimédia, ainsi que le 
Cycles de vie et carrières dans les métiers des TIC : une perspective de genre

caractère sexué des productions : logiciels, interfaces, pages web, etc. (Balka et Smith, 2000 ; Claeys et Spee, 2005 ; Cohoon et Aspray, 2006 ; Collet, 2006 ; Merete, 2003).

Diverses institutions internationales ont également publié des rapports dressant un état des connaissances dans ce domaine (European Commission, 2008 ; OECD, 2007). La Commission européenne a en outre soutenu la collecte et la mise en réseau de bonnes pratiques visant à améliorer la situation des femmes dans les métiers des TIC ${ }^{1}$.

Le groupe professionnel concerné par ces recherches n'est pas homogène. Alors que certaines recherches privilégient les métiers très qualifiés de l'ingénierie informatique, d'autres incluent un spectre plus large de qualifications, d'autres encore intègrent les métiers émergents du multimédia et des services sur internet. C'est pourquoi il s'avère nécessaire de préciser d'emblée ce qu'on entend ici par "métiers des TIC », car cette appellation générique recouvre plusieurs catégories de professions (Valenduc et al., 2004a) :

- Le noyau traditionnel des métiers de l'informatique, c'est-à-dire la conception et l'ingénierie des systèmes d'information et des logiciels, l'ingénierie des réseaux, le développement d'applications, l'analyse et la programmation, les tests de qualité, le paramétrage, la maintenance matérielle et logicielle, l'assistance aux utilisateurs, etc.

- Les nouveaux métiers de l'internet et du multimédia, qui combinent des compétences en informatique et en réseaux avec des compétences dans le domaine de la communication ou des arts graphiques.

- Les métiers liés à des domaines d'applications spécifiques, par exemple l'informatique médicale, l'informatique scientifique, les plateformes de commerce électronique, les progiciels de gestion intégrée (ERP), de gestion de la chaîne logistique (SCM) ou de gestion de la clientèle (CRM), etc.

Comme les frontières entre les métiers des TIC et certains métiers d'utilisateurs spécialisés ont tendance à se brouiller, il faut également prendre en compte la présence, dans les métiers des TIC, de professionnels en provenance d'autres horizons :

1. Ces initiatives sont accessibles via le portail www.ictwomendirectory.eu 
- Des ingénieurs non informaticiens, qui peuvent exercer des métiers des TIC dans leur branche industrielle (chimie, métallurgie, électricité, construction, etc.), ou des ingénieurs de gestion, spécialisés dans le management des systèmes d'information.

- Des « utilisateurs concepteurs », par exemple des bibliothécaires qui conçoivent et gèrent des systèmes d'information, des pédagogues qui conçoivent et gèrent des plateformes d'e-learning, des architectes qui conçoivent des systèmes de modélisation des bâtiments, etc.

D'un groupe de métiers à l'autre, la proportion de femmes peut varier considérablement. C'est dans les métiers du noyau traditionnel, c'est-à-dire l'informatique au sens étroit du terme, qu'elle est la plus faible.

Cet article repose sur diverses recherches qui ont été réalisées à la Fondation Travail-Université (Namur, Belgique) au cours des dernières années. II s'agit, en premier lieu, de deux projets européens: le projet WWW-ICT (Widening Women's Work in Information and Communication Technology), déjà cité, qui concernait spécifiquement la place des femmes dans les professions des TIC (Valenduc et al., 2004a), ainsi que le projet WORKS (Work Organisation and Restructuring in the Knowledge Society), qui comportait plusieurs études de cas sur le secteur des services informatiques (Valenduc et al., 2008) et qui s'est également intéressé aux carrières dans les métiers intensifs en connaissances (Valenduc et al., 2009). Quelques données et illustrations seront également empruntées à une troisième recherche, réalisée en Wallonie (Vendramin, 2004). La notion de «cycle de vie » provient d'une conférence tenue à l'Internet Interdisciplinary Institute (IN3) de l'Université ouverte de Catalogne, en novembre 2010, dont plusieurs contributions figurent parmi les références bibliographiques.

Dans une tentative de synthèse de ces différentes approches, le projet WWW-ICT a relevé quatre catégories d'hypothèses qui sont habituellement avancées pour tenter d'expliquer les disparités de genre dans les professions des TIC (Valenduc et al., 2004a). Ces quatre catégories d'hypothèses explicatives concernent :

- Les déséquilibres dans l'éducation et la formation: s'il y a peu de femmes dans ces métiers, c'est parce qu'elles ne sont pas orientées vers les choix d'études qui y mènent, à cause d'une image de l'informatique peu attractive pour les femmes, dès l'enfance et l'adolescence.

- Des conditions de travail qui défavorisent les femmes: les emplois des TIC ont la réputation d'exiger de longues heures de travail, des 
Cycles de vie et carrières dans les métiers des TIC : une perspective de genre

horaires imprévisibles, une disponibilité permanente, une flexibilité peu compatible avec des contraintes familiales.

- Des carrières professionnelles qui favorisent les hommes: la progression professionnelle repose avant tout sur des règles du jeu qui sont définies par des hommes et pour des hommes. Les interruptions de carrière et les réductions volontaires du temps de travail sont malvenues. Les femmes sont certes nombreuses dans les fonctions de chef de projet mais grimpent rarement plus haut dans la hiérarchie.

- $\quad$ - Des facteurs culturels qui renforcent l'image masculine des TIC : les stéréotypes relatifs à la culture professionnelle de l'informatique sont un mélange de la culture de domination du programmeur et de la culture alternative du pionnier. Ces valeurs et ces modèles de comportement conviennent mieux aux hommes qu'aux femmes.

Ces hypothèses sont spécifiques aux TIC et se distinguent, en partie, des hypothèses relatives à l'ensemble des professions scientifiques et techniques (Alaluf et al., 2004). Cette démarcation concerne principalement les questions liées aux conditions de travail et aux facteurs culturels.

Selon les résultats de WWW-ICT, aucune de ces hypothèses explicatives ne peut, à elle seule, expliquer les écarts qui existent entre les hommes et les femmes dans les parcours de formation et les parcours professionnels dans les TIC. De plus, le caractère apparemment universel de ces hypothèses, c'est-àdire le fait qu'elles sont formulées sans référence à des contextes nationaux ou socioéconomiques particuliers, contraste avec des situations très différenciées d'un pays à l'autre en Europe, aussi bien en termes de présence des femmes dans les filières de formation aux TIC que sur le plan des disparités sur le marché du travail.

Par rapport aux hypothèses explicatives habituellement avancées dans la littérature, les résultats de WWW-ICT apportent quelques surprises (Vendramin, 2005).

Tout d'abord, les femmes qui occupent des métiers des TIC ne semblent pas avoir de problèmes particuliers avec la technologie. Elles décrivent l'univers des TIC comme créatif, stimulant, fascinant et source de satisfactions. L'orientation professionnelle n'est pas fortement influencée par la famille. Plus particulièrement, les pères intéressés par l'ingénierie et les mathématiques ne jouent pas de rôle déterminant; parmi les 107 femmes rencontrées lors des entretiens biographiques dans six pays, très peu avaient un père exerçant un métier technologique. 
Ensuite, bien qu'il ressorte des études de cas d'entreprises et des entretiens biographiques que les conditions de travail sont relativement exigeantes dans ce secteur (particulièrement le temps de travail), il n'apparaît pas que ceci soit la principale variable qui explique la présence limitée des femmes. Souvent, les femmes acceptent ces conditions de travail en échange d'une grande autonomie dans la gestion de leur temps de travail. Les chercheurs de WWWICT ont rencontré très peu de cas de femmes qui avaient quitté leur emploi pour ce type de raisons, de même que peu de femmes insatisfaites par leur travail actuel dans les TIC.

Enfin, il n'y a pas de chemin unique qui conduit les femmes aux métiers des TIC, contrairement aux parcours masculins, fortement déterminés par la formation initiale. Parmi les femmes, on trouve des diplômées des filières scientifiques et techniques mais aussi des filières artistiques, du journalisme, de l'économie et des sciences humaines. De plus, un nombre significatif de femmes interviewées sont entrées dans ces métiers à partir d'autres postes occupés dans la même entreprise, ou bien à partir d'autres domaines d'activités, ou encore à l'issue d'une reconversion professionnelle.

Ces résultats suggèrent que, pour comprendre les disparités de genre dans les métiers des TIC, il est nécessaire de s'orienter vers une approche en termes de parcours professionnels ou, mieux, en termes de cycle de vie - car la notion de cycle de vie intègre à la fois des éléments du parcours professionnel et du parcours personnel en dehors du travail. Cette approche permet de proposer une articulation entre des résultats de recherche portant tantôt sur l'éducation et la formation, tantôt sur le recrutement, tantôt sur les conditions de travail, tantôt sur les carrières et la mobilité professionnelle.

\section{Deux paradoxes}

Deux grands paradoxes caractérisent aujourd'hui la situation des femmes dans les métiers des TIC. D'abord, malgré de nombreuses campagnes de sensibilisation, la position des femmes dans les professions des TIC ne s'est pas améliorée, ni en Belgique, ni dans la plupart des autres pays européens. Ensuite, il y a proportionnellement plus de femmes dans les métiers des TIC que dans les études supérieures qui mènent à ces métiers; elles sont donc nombreuses à venir d'autres horizons. 
Cycles de vie et carrières dans les métiers des TIC :

une perspective de genre

\subsection{Une situation qui se détériore}

La prise de conscience des disparités entre hommes et femmes dans les métiers des TIC n'est pas récente. Le besoin d'attirer davantage de femmes vers ces métiers a été exprimé clairement par les entreprises et les institutions de formation au moment du boom de la "nouvelle économie", il y a une douzaine d'années. Diverses associations qui militent en faveur de l'égalité professionnelle entre les femmes et les hommes se sont emparées de ce thème. En Belgique, le projet «ADA - femmes et informatique » a réalisé, de 2000 à 2007, un remarquable travail de sensibilisation et de mise en réseau ${ }^{2}$. Des campagnes ont également été menées par les pouvoirs publics dans de nombreux pays et par la Commission européenne elle-même, avec l'appui de certaines grandes entreprises du secteur des TIC (Commission européenne, 2006). Diverses plateformes nationales ou internationales se sont constituées, dont le European Centre for Women and Technology, qui bénéficie du soutien de la Commission européenne et d'industriels du secteur des TIC.

$\mathrm{Si}$ on remonte loin dans le temps, c'est-à-dire dans les années 1980, l'informatique était présentée comme une discipline technologique particulièrement ouverte aux femmes. À l'université, les étudiantes en informatique étaient nombreuses : 35 à $40 \%$, contre moins de 10\% actuellement (Collet, 2004). Cette époque est révolue.

$\mathrm{Au}$ cours de la dernière décennie, l'emploi des femmes dans les TIC a augmenté plus lentement que l'emploi des hommes, si bien que les écarts se sont accentués (tableau 1).

2. Les archives des publications de ce projet sont consultables sur www.ada-online.org. 
Gérard VALENDUC

\section{Tableau 1 - Pourcentage de la population active occupée qui travaille dans des métiers des TIC, par sexe}

Emploi dans les catégories professionnelles ISCO 213 (spécialistes en informatique) et ISCO 312 (techniciens en informatique), en \% de la population d'hommes ou de femmes en emploi dans chaque pays

\begin{tabular}{|c|c|c|c|c|c|c|}
\hline & \multicolumn{2}{|c|}{2001} & \multicolumn{2}{c|}{2006} & \multicolumn{2}{c|}{$\begin{array}{c}\text { Différence en points de } \\
\text { pourcentage }\end{array}$} \\
\hline & Femmes & Hommes & Femmes & Hommes & Femmes & Hommes \\
\hline EU25 & 0.7 & 2.3 & 0.7 & 2.6 & 0.0 & 0.3 \\
\hline Belgique & 0.5 & 2.6 & 0.6 & 2.8 & 0.1 & 0.2 \\
\hline Allemagne & 0.7 & 2.5 & 0.8 & 2.9 & 0.0 & 0.2 \\
\hline Danemark & 0.9 & 3.2 & 1.1 & 3.8 & 0.2 & 0.6 \\
\hline Espagne & 0.6 & 1.4 & 0.6 & 2.0 & 0.0 & 0.6 \\
\hline France & 0.7 & 2.8 & 0.7 & 2.7 & 0.0 & -0.1 \\
\hline Hongrie & 0.6 & 1.6 & 0.5 & 2.4 & -0.1 & 0.8 \\
\hline Italie & 0.6 & 1.4 & 0.6 & 1.9 & 0.0 & 0.5 \\
\hline Suède & 1.4 & 5.2 & 1.5 & 4.9 & 0.1 & -0.3 \\
\hline UK & 1.0 & 3.5 & 0.8 & 3.3 & -0.2 & -0.2 \\
\hline
\end{tabular}

Source : EUROSTAT, Statistics in focus, $n^{\circ} 119,2007$.

Données de l'enquête européenne sur les forces de travail, 2001 et 2006.

Ce tableau montre que, parmi les femmes en emploi, la proportion de celles qui occupent un métier des TIC n'a quasiment pas augmenté de 2001 à 2006, alors que la proportion d'hommes a augmenté de manière significative - à l'exception du cas suédois.

En Belgique, dans le secteur du conseil et des services informatiques, l'emploi féminin (tous métiers confondus) a augmenté de $25 \%$ entre 2000 et 2009, alors que l'emploi masculin a augmenté de 34\%. Dans les statistiques sur les professions (tous secteurs confondus), la proportion de femmes parmi les spécialistes des TIC est passée de 18\% en 1997 à 16\% en 2006.

En Belgique toujours, la quantité et la proportion de femmes dans l'enseignement supérieur (universités et hautes écoles) dans le domaine des TIC est en diminution, comme dans la plupart des pays européens. Par rapport 
Cycles de vie et carrières dans les métiers des TIC :

une perspective de genre

à l'année académique 2004-2005, la proportion d'étudiantes en 2007-2008 a régressé dans toutes les filières TIC de l'enseignement supérieur, à l'exception des ingénieurs civils en informatique. Le cas des baccalauréats ${ }^{3}$ en infographie et multimédia, qui représentent aujourd'hui la catégorie la plus importante parmi les baccalauréats en TIC, est révélateur. II s'agit d'un domaine nouveau, qui combine des compétences en informatique et en communication, où l'on s'attendrait à trouver davantage de femmes. C'est le cas : elles sont aujourd'hui $21 \%$ parmi les étudiants de ces filières ... mais elles étaient $24 \%$ quatre ans plus tôt. D'une manière générale, la diminution de la proportion d'étudiantes dans le domaine des TIC va à contre-sens d'une tendance plus large d'augmentation de la proportion d'étudiantes dans les disciplines scientifiques et techniques.

\subsection{La diversité des parcours de formation des femmes}

Le deuxième paradoxe réside dans le fait que la proportion de femmes occupée dans les métiers des TIC, que ce soit dans le secteur TIC lui-même ou dans des entreprises utilisatrices, est nettement plus élevée que la proportion de femmes dans les études supérieures qui conduisent à ces métiers. En Belgique (2008), il y a $16 \%$ de femmes dans la catégorie professionnelle des spécialistes des TIC (ISCO 213 dans la nomenclature des professions), contre à peine $8 \%$ parmi les diplômés des filières correspondantes dans les universités et hautes écoles. L'écart est semblable dans des pays comme la France, l'Espagne ou la Grande-Bretagne.

Ce constat laisse présumer qu'un grand nombre de femmes qui occupent actuellement un métier des TIC y sont arrivées après une formation initiale dans d'autres domaines, éventuellement suivie d'une formation complémentaire dans les TIC. Cette hypothèse est confirmée par diverses données sur la présence des femmes dans les filières TIC de l'enseignement de promotion sociale et de la formation professionnelle continuée (centres de compétences TIC, FOREM, Bruxelles Formation, IFAPME, CEFORA, etc.). Bien que toujours largement minoritaires, les femmes y sont toutefois plus nombreuses que dans la formation initiale. Par exemple, en 2007-2008, les femmes représentaient $25 \%$ des diplômés TIC dans l'enseignement de promotion sociale en Belgique francophone.

3. Il s'agit du premier cycle de l'enseignement supérieur à l'issue de la réforme de Bologne (licence en France, bachelor en anglais). 
Par conséquent, de nombreuses professionnelles des TIC ont une double qualification: celle de leur formation initiale et celle de leur formation complémentaire. II n'est pas rare de rencontrer des femmes qui ont un diplôme universitaire en dehors des TIC, complété par une formation spécialisée dans un domaine des TIC. Les professionnels masculins sont plus nombreux à avoir une formation initiale dans le domaine des TIC, même quand ils ont aussi une formation complémentaire.

\section{De nouvelles tendances dans les métiers des TIC}

\subsection{Une nouvelle division internationale du travail}

La nouvelle division internationale du travail dans le secteur du logiciel et des services informatiques exerce une influence importante sur l'évolution actuelle des métiers des TIC. II s'agit de changements qui concernent à la fois la division géographique, technique et sociale du travail, à toutes les étapes de la " chaîne de valeur » des services informatiques (voir graphique $\mathrm{n}^{\circ} 1$ ). 
Cycles de vie et carrières dans les métiers des TIC : une perspective de genre

Graphique 1

La chaîne de valeur des services TIC

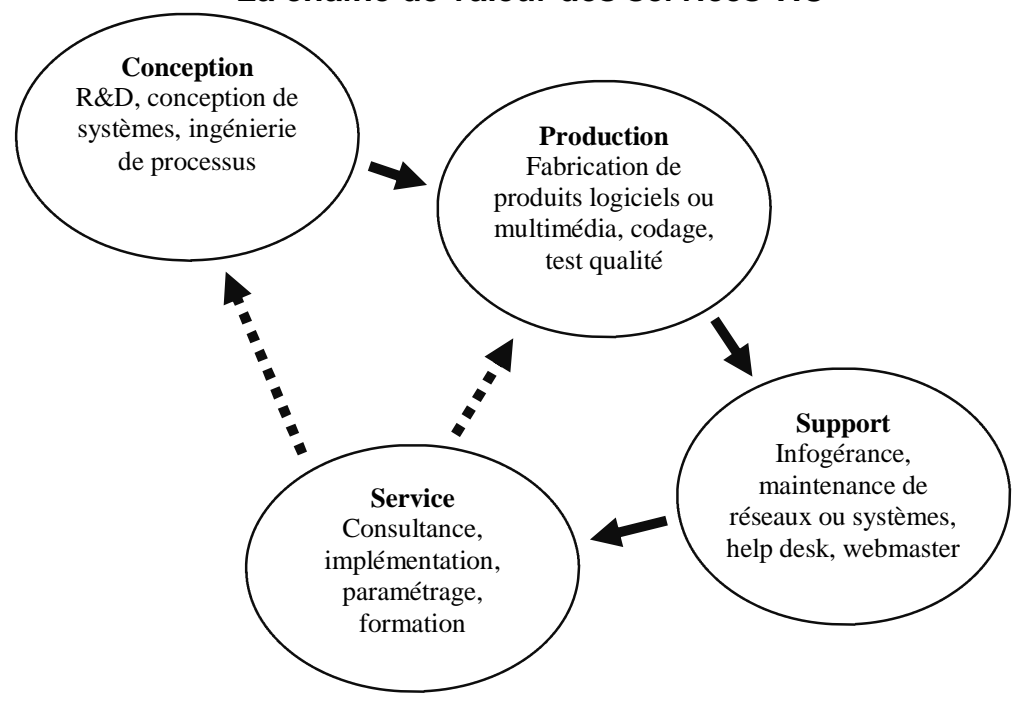

L'hypothèse avancée ici est que la nouvelle division du travail, liée aux changements dans la chaîne de valeur des services informatiques, a des effets différenciés pour les hommes et pour les femmes

La division technique du travail est caractérisée par une importance croissante de la codification des connaissances et des procédures, qui conduit à une standardisation des tâches, notamment dans la programmation, l'analyse et le contrôle qualité. Cela favorise la sous-traitance locale ou internationale. La codification a une contrepartie : les tâches de gestion de projet, de marketing, de communication, de relations avec les clients prennent une importance croissante.

La division géographique du travail repose sur l'externalisation lointaine (offshoring) de tâches préalablement codifiées et standardisées, ainsi que de services faciles à délocaliser grâce aux nouvelles technologies de réseau : le help desk, l'infogérance, le codage, la maintenance à distance, l'exploitation de ressources matérielles partagées (grid computing). La division géographique du travail touche aussi les activités de R\&D. Les grands laboratoires européens s'implantent en Inde, en Asie du Sud-Est ou en Californie, pour développer la veille technologique et établir des partenariats. En Europe, le rapprochement 
entre la recherche et le marché donne naissance à des laboratoires hybrides, à l'interface entre science et industrie.

La division sociale du travail se caractérise par des changements dans les statuts respectifs des différents métiers, selon qu'ils sont orientés vers la technique, le management, la communication ou la relation commerciale. Tous les segments de la chaîne de valeur sont affectés par ces changements. Si le service a tendance à se rapprocher des clients, la production et le support sont largement externalisés et internationalisés.

Les emplois des hommes et des femmes ne sont pas affectés de la même manière par les différentes formes d'externalisation. Les délocalisations lointaines touchent surtout des bastions masculins, comme la programmation, la maintenance à distance, l'exploitation de ressources informatiques partagées. Les nouvelles formes de division technique du travail renforcent la ségrégation entre les rôles attribués aux hommes et aux femmes dans les tâches techniques, commerciales, relationnelles ou managériales. Quant à la soustraitance, elle est fréquente dans les métiers du web et du multimédia, où les femmes sont plus présentes, ainsi que dans les petites entreprises.

Du point de vue des métiers, ces transformations structurelles des services TIC entraînent de nombreux changements. Parmi toutes les transformations mises en évidence par les études de cas du projet WORKS (Valenduc et al., 2008), trois tendances particulières ont été retenues ici, parce qu'elles ont des effets différenciés sur les hommes et les femmes: l'élargissement du portefeuille de compétences, la diversification des modèles de carrière et l'évolution des cultures professionnelles.

\subsection{L'élargissement du portefeuille de compétences}

Une tendance commune à tous les métiers des TIC est l'élargissement du portefeuille de compétences au-delà des qualifications qui constituent le cœur du métier (Ramioul \& De Vroom, 2009).

Dans la nouvelle division du travail, les compétences émergentes en Europe concernent la R\&D, la sécurité, les interfaces homme-machine, la qualité des logiciels, la gestion d'équipes, la gestion de projets, la formation et l'assistance aux utilisateurs. Les compétences de gestion, ainsi que les compétences non techniques (soft skills) prennent une importance croissante, de même que certaines compétences techniques très pointues. Les résultats du projet européen WORKS permettent de préciser quels sont les domaines de 
Cycles de vie et carrières dans les métiers des TIC : une perspective de genre

compétences qui sont affectés par ces changements (Valenduc et al., 2008, p. 87) :

- Les compétences méthodologiques, notamment dans le domaine de l'analyse fonctionnelle, des méthodologies de développement de logiciels, de l'assurance qualité.

- Les compétences de gestion de projet, qui comprennent à la fois des compétences techniques (planification et spécifications fonctionnelles), économiques (contrôle des budgets) et humaines (gestion d'équipes) ; l'externalisation lointaine de la programmation renforce l'importance de la gestion de projets en Europe.

- Les compétences de communication avec les clients: capacité de comprendre leurs besoins ou leurs problèmes, de dialoguer tout au long du projet, de traduire des demandes complexes en spécifications précises, de se familiariser rapidement avec le secteur d'activité du client et la culture organisationnelle de son entreprise ou institution ; les compétences linguistiques font partie de ces compétences de communication.

- Les compétences d'interaction sociale : l'assertivité, la capacité de travailler en équipe, l'autonomie dans l'exécution des tâches, l'intégration dans une culture professionnelle particulière.

Toutefois, la majorité des professionnels des TIC - hommes ou femmes interviewés dans les différents pays partenaires du projet WORKS n'établissent pas spontanément de lien de cause à effet entre la mondialisation et l'évolution de leur portefeuille de compétences. Ils considèrent souvent que cette évolution est due à la dynamique interne de leur profession. Ils s'inquiètent cependant des tensions croissantes entre, d'une part, la créativité ou l'innovation, et d'autre part, la pression directe du marché et l'accélération des activités, se demandant si ces tensions seront soutenables à long terme

La division entre compétences techniques et compétences relationnelles est fortement marquée par les stéréotypes de genre : aux hommes la technique, aux femmes la communication. Dans l'informatique et le multimédia, il y a de nombreuses femmes chefs de projet, mais peu de programmeuses. Les compétences relationnelles sont plus souvent attribuées aux femmes qu'aux hommes, mais elles sont mal reconnues dans les carrières et peu valorisées dans les rémunérations.

Le fait que de nombreuses femmes soient rentrées dans un métier des TIC après une autre formation initiale leur confère ici un atout, celui d'avoir souvent un double profil de qualifications, combinant une formation technique et une 
formation non technique. L'élargissement du portefeuille de compétences est donc généralement favorable aux femmes, mais elles peuvent se retrouver enfermées dans des rôles qui leur sont attribués par les hommes.

\subsection{La diversification des modèles de carrière}

Les transformations de la chaîne de valeur, et plus particulièrement l'importance stratégique croissante des phases de conception, d'une part, et de service à la clientèle, d'autre part, exercent une influence sur les modèles de carrière des hommes et des femmes dans les métiers des TIC.

Le débat sur les carrières dans les TIC est marqué par l'opposition entre les carrières organisationnelles, réputées appartenir au passé, et les carrières nomades ou carrières sans attache (boundaryless careers), censées préfigurer les nouvelles formes de travail (Cadin et al., 2003 ; Guerrero, 2001). Cette opposition est très présente dans la littérature sur les carrières des « travailleurs du savoir " (Bouchez, 2004; Huws, 2007). Elle doit être relativisée car les modèles de carrière se sont récemment diversifiés (Valenduc et al., 2009) :

- Les carrières organisationnelles, c'est-à-dire où les règles de progression sont essentiellement fixées par l'entreprise ou l'institution, se débarrassent de leur image bureaucratique. Le modèle hiérarchique pyramidal évolue vers un modèle plus fonctionnel.

- À côté des carrières hiérarchiques, il y a aujourd'hui de plus en plus de carrières organisationnelles techniques, où la progression repose sur une expertise croissante, reconnue à l'intérieur et à l'extérieur de l'organisation.

- Des carrières «multi-organisationnelles » apparaissent. Elles sont caractérisées par une certaine mobilité des individus d'une entreprise à l'autre, jusqu'à ce qu'ils aient trouvé l'organisation qui leur convient le mieux.

- Les carrières nomades - c'est-à-dire construites volontairement par l'individu sur base de ses propres compétences, sans attache à une organisation - sont souvent limitées à certains métiers, notamment dans le multimédia (Krings, 2007 ; Tremblay, 2003), ou à certaines zones métropolitaines, comme Londres, Paris ou Milan.

- Un nouveau type de carrière se répand depuis peu: des carrières fragmentées, caractérisées par une succession de choix contraints, dus à des licenciements, à des fermetures ou délocalisations 
Cycles de vie et carrières dans les métiers des TIC :

une perspective de genre

d'entreprises, ou à une instabilité devenue chronique. II s'agit, en quelque sorte, d'un nomadisme forcé.

Pour les femmes, cette diversification des modèles de carrière a des effets contrastés (Bender et al., 2001 ; Valcourt \& Tolbert, 2003).

Dans les carrières hiérarchiques, la persistance du «plafond de verre » empêche souvent les femmes de dépasser certains niveaux - par exemple, celui de chef de projet. Dans le secteur des TIC, les systèmes de progression sont souvent opaques, au désavantage des femmes. Quant aux carrières organisationnelles techniques, elles restent largement verrouillées par les hommes, qui bénéficient d'un effet de cooptation par leurs pairs, notamment au sein des comités d'experts et des comités techniques des organisations professionnelles. Dans les carrières multi-organisationnelles, les femmes ne sont pas moins mobiles que les hommes, contrairement à une idée reçue, mais leurs choix de mobilité suivent des motivations différentes, qui combinent, plus souvent que chez les hommes, des objectifs professionnels et des objectifs de qualité de vie.

Du point de vue du genre, les carrières nomades font l'objet d'appréciations controversées, qui renvoient au bilan des avantages et des inconvénients de l'autonomie et de la prise de risques individuels - un bilan qui peut évoluer avec l'âge et la situation personnelle.

Les carrières fragmentées sont fréquentes chez les femmes, qui sont beaucoup plus exposées que les hommes aux conséquences des interruptions de carrière, notamment pour la maternité et l'éducation des jeunes enfants, ainsi qu'aux difficultés de trouver l'équilibre entre travail et vie privée ou familiale. Ici, c'est l'inégale répartition des rôles masculins et féminins dans la société en général, et dans la famille en particulier, qui est défavorable aux carrières des femmes dans des métiers souvent exigeants en termes de disponibilité et d'imprévus.

\subsection{L'évolution de la culture professionnelle}

Les métiers des TIC se caractérisent par une culture professionnelle spécifique, dont les traits peuvent se dessiner à partir d'une analyse de leurs pratiques de travail (Vendramin, 2007). Cependant, cette culture professionnelle spécifique donne parfois lieu à des images caricaturales, notamment dans les films de fiction, et à des représentations stéréotypées qui défavorisent la profession (Collet, 2006). Ces images caricaturales sont en porte-à-faux avec la nouvelle réalité du travail dans les métiers des TIC. 
Les professionnels des TIC ont très souvent une forte implication personnelle dans leur travail et une disposition à apprendre tout au long de la vie. Ils s'identifient davantage à un métier ou à un domaine spécialisé qu'à une entreprise ou une institution. Ils attachent de l'importance à une bonne qualité de relations interpersonnelles au travail, mais dans des petits groupes, liés à des projets ou à des spécialisations. Ils développent des communautés de pratiques ou d'autres outils d'échange en ligne. Ils cherchent souvent dans leur travail une dimension expressive, d'accomplissement personnel ou d'intégration dans un réseau de relation - ce qui est assez éloigné du stéréotype de l'informaticien fanatique et asocial. Cependant, ils pressentent des menaces sur le caractère créatif de leur travail, à cause de la codification des connaissances et de la pression croissante des impératifs commerciaux. La réorganisation de la chaîne de valeur confronte les spécialistes de la technologie avec d'autres cultures professionnelles, provenant de la sphère économique et commerciale ou de l'univers de la création multimédia.

Si la dimension expressive du travail est commune aux hommes et aux femmes, d'autres aspects de la culture professionnelle sont beaucoup plus problématiques pour les femmes. La pression des entreprises pour l'autoformation en dehors du travail est défavorable aux femmes, qui disposent de beaucoup moins de temps libre que les hommes. Le modèle familial traditionnel de l'homme "gagne-pain » est encore prédominant dans ce milieu professionnel et relègue au second plan le besoin des femmes de faire carrière.

La culture d'entreprise du secteur des TIC est aveugle par rapport au genre, dans le sens où elle ne reconnaît pas les situations différenciées des hommes et des femmes. Le message adressé aux femmes est: " faites comme les hommes " (Krings, 2007). La culture de métier, qui exalte pourtant la flexibilité, est peu ouverte à des arrangements en termes de temps de travail qui pourraient répondre aux besoins des femmes. Les femmes sont bien plus exposées que les hommes aux renoncements : renoncement à la carrière, au développement d'une famille, à la mobilité.

L'évolution des portefeuilles de compétences, des modèles de carrière et des cultures professionnelles présente donc un bilan contrasté sur le plan du genre. Dans le secteur des TIC, la culture d'entreprise est souvent un mélange instable de comportements novateurs et conservateurs.

\section{Les entrées et sorties des femmes dans les métiers des TIC}

La diversité des chemins d'entrée des femmes dans les métiers des TIC a déjà été mise en évidence. À côté des entrées directes, via un diplôme de 
Cycles de vie et carrières dans les métiers des TIC : une perspective de genre

l'enseignement supérieur dans le domaine des TIC, il y a beaucoup d'entrées indirectes, via d'autres diplômes complétés par une formation TIC, certifiée ou non. Cette situation est très fréquente dans les métiers de l'internet et du multimédia, ainsi que dans les métiers liés à des domaines d'application spécifiques. Le fait d'avoir une double formation, voire un double diplôme, permet des possibilités de carrière plus ouvertes en termes de mobilité horizontale ou verticale, mais souvent en quittant les métiers des TIC proprement dits.

En plus des entrées directes et indirectes, il y a aussi des entrées postposées, qui s'effectuent à un stade plus avancé de la vie professionnelle, suite à une période de chômage, à une interruption d'activité professionnelle ou à une reconversion volontaire. Les dispositifs de formation professionnelle qualifiante jouent ici un rôle essentiel. Les métiers des TIC s'avèrent alors attractifs pour de nombreuses femmes en situation de réinsertion, paradoxalement davantage que pour de jeunes étudiantes. II se peut que la motivation du contenu et du contexte de travail soit à ce stade prédominante par rapport à la perspective de carrière. Ce constat repose cependant sur des méthodes de recherche qualitatives, fondées sur des études de cas d'entreprises ou des entretiens biographiques. Les données quantitatives sur les chemins d'entrée ou de réinsertion dans les métiers des TIC sont lacunaires.

Les sorties du métier sont également un phénomène important, appelé par certains auteurs "tuyau percé » (leaky pipeline), à tel point que les abandons de carrière commencent à inquiéter certains industriels des TIC et à susciter des politiques de rétention spécifiques aux femmes (European Commission, 2006). Dans une enquête menée en 2004 en Belgique francophone, un quart des répondantes occupant un métier des TIC pensaient qu'elles n'allaient pas faire toute leur carrière dans ces métiers. Parmi les diplômés TIC qui avaient quitté les métiers des TIC, un tiers étaient des femmes. Les principaux obstacles perçus par les femmes sont l'opacité des règles de progression, le plafond de verre, la difficulté de se faire reconnaître par des pairs majoritairement masculins, ainsi que les inégalités de traitement dont elles font l'objet (Vendramin, 2004).

\section{Conclusions}

La compréhension des inégalités entre les hommes et les femmes dans les métiers des TIC est un thème de recherche qui est toujours en chantier. Les hypothèses explicatives trop simples ou trop univoques sont insatisfaisantes, 


\section{Gérard VALENDUC}

mais il ne suffit pas non plus d'affirmer que la problématique est complexe et multidimensionnelle pour y mettre un point final. L'absence de succès dans la réduction des inégalités n'est pas seulement due à un déficit de compréhension du processus social qui génère ces inégalités. Elle est aussi liée à la difficulté de concevoir et de mettre en œuvre des campagnes de sensibilisation et des mesures politiques efficaces, au-delà des sempiternelles collectes de "bonnes pratiques" dont la Commission européenne est particulièrement friande.

C'est pourquoi l'approche en termes de cycle de vie, développée par le programme de recherche «Gender and ICT» de l'Internet Interdisciplinary Institute ${ }^{4}$, s'avère particulièrement pertinente. Cette approche permet de suivre les trajectoires des femmes depuis les premiers niveaux de l'éducation scolaire et de son contexte familial et social, jusqu'aux développements de la carrière professionnelle et du parcours de vie. Elle présente plusieurs avantages. Tout d'abord, elle permet d'identifier les principaux facteurs qui façonnent l'engagement des femmes dans les TIC, à différents moments de leur vie, et d'examiner s'ils ont un effet cumulatif ou s'ils présentent des synergies particulières. Ensuite, l'approche en termes de cycle de vie peut mettre en évidence les moments de transition importants dans la vie des femmes, ainsi que les moments où se manifestent l'engouement ou l'usure, l'adhésion ou l'abandon. II s'agit notamment d'expliquer pourquoi et comment les carrières des femmes sont influencées non seulement par le monde de l'enseignement et le monde de l'entreprise, mais aussi par les rapports de genre dans la sphère domestique. Enfin, cette approche permet d'accorder une meilleure reconnaissance aux choix et aux décisions des femmes elles-mêmes, ainsi qu'à la façon dont elles font face aux situations changeantes de leur environnement personnel et professionnel.

Le concept de cycle de vie ouvre non seulement de meilleures perspectives analytiques, mais aussi de meilleures opportunités pour concevoir des lignes d'intervention, en ciblant des domaines spécifiques ou des moments particuliers du cycle de vie.

La problématique des disparités de genre dans les métiers des TIC est donc loin d'être résolue. Si on lui accorde tant d'importance, c'est notamment parce que ces métiers influencent l'organisation du travail et l'organisation de la société. Les inégalités professionnelles y sont aussi des inégalités en termes de pouvoir dans la société.

\footnotetext{
${ }^{4}$ II s'agit d'un Institut fondé par Manuel Castells au sein de l'Université ouverte de Catalogne : http://in3.uoc.edu
} 
Cycles de vie et carrières dans les métiers des TIC :

une perspective de genre

\section{Références bibliographiques}

ALALUF M., N. IMATOUCHAN, P. MARAGE, S. PAHAUT, R. SANVURA, A. VALKENEERS, 2004, Les filles face aux études scientifiques (vol. I) - Les femmes et les professions scientifiques (vol. II), Rapports NEWTONIA, Bruxelles, Presses de l'ULB.

BALKA E., R. SMITH (eds), 2000, Women, Work and Computerization, Boston, Kluwer Academic Publishers.

BARKER L., J. COHOON, L. SANDERS, 2010, « Strategy Trumps Money : Recruiting Undergraduate Women into Computing », in IEEE Computer Magazine, 43/6, NY, pp. 82-86

BENDER, A-F., F. PIGEYRE, V. DE SAINT-GINIEZ, 2001, "Les femmes dans la société de l'information : opportunités et risques de nouvelles carrières ", ans les Actes du 21 ème congrès de l'AGRH La GRH dans la société de l'information, Université de Liège, septembre 2001, pp. 891-909.

BOUCHEZ J.P., 2004, Les nouveaux travailleurs du savoir, Paris, Éditions d'Organisation.

CADIN L., A.F. BENDER, V. DE SAINT-GINIEZ, 2003, Carrières nomades : les enseignements d'une comparaison internationale, Paris, Vuibert.

CASTAÑO C., 2008, Género y TIC : presencia, posición y políticas, Barcelona, Editorial UOC.

CASTAÑO C., M. SÁINZ, A.M. GONZÁLEZ, R. PALMÉN, J. MÜLLER, 2009, Gender and innovation for corporate change in ICT : culture as continuity or change for women workers?, Barcelona, IN3 Working Papers Series, Open University of Catalunya.

CLAEYS L., SPEE S., 2005, Gender in de netwerkmaatschappij : een virtuele illusie of reële kansen ?, Antwerpen, Steunpunt Gelijkekansenbeleid.

COHOON J. and ASPRAY W. (eds), 2006, Women and Information Technology : Research on Under-Representation, MIT Press.

COHOON J., 2009, "Scarcity of Women in Computing : Attracting and Retaining Women Undergraduates in Computer Science ", in Encyclopedia of Computer Science and Engineering, John Wiley \& Sons, Inc. Hoboken, NY, pp. 3099-3122 
COLLET I., "La disparition des filles dans les études d'informatique : les conséquences d'un changement de représentation ", dans Carrefour de l'éducation, Paris, 1/2004 (n¹7), p. 42-56.

COLLET I., L'informatique a-t-elle un sexe ?, L'Harmattan, Paris, 2006.

EUROPEAN COMMISSION, 2006, Best practices for even gender distribution in the 25 Member States in the domain of ICT, DG Information Society and Media, December 2006.

EUROPEAN COMMISSION, 2008, Women in ICT : status and the way ahead, DG Information Society and Media, January 2008.

EUROSTAT, 2009, Gender differences in the use of computers and the Internet, Statistics in Focus 119/2007.

GRAS-VELAZQUEZ A., A. JOYCE, M. DEBRY, Women and ICT. Why are girls still not attracted to ICT studies and careers?, Brussels, White Paper of EUN European Schoolnet.

GUERRERO S., 2001, « La contribution des TIC au développement des nouvelles carrières ", dans les Actes du 21ème congrès de l'AGRH La GRH dans la société de l'information, Université de Liège, septembre 2001, pp. 449-464.

HUWS U., 2007, "The spark in the engine : creative workers in a global economy ", in Work organization, labour and globalisation, vol.1(1), London, Merlin Press, pp. 1-12

KIRKUP G., 2010, « Vocational education and training for ICT employment : preparing women for work », Conference Women and ICT through the lifecycle, 8-10 November 2010, Barcelona, Internet Interdisciplinary Institute.

KRINGS B.J., 2007, « Make like a man : the demands of creative work, gender and everyday life ", in Work organisation, labour and globalisation, vol.1(1), London, Merlin Press, pp. 89-107

MERETE L. (ed.), 2003, He, She and IT revisited-New Perspectives on Gender in the Information Society, Trondheim, Gyldendal Akademisk.

OECD, 2007, ICTs and Gender, OECD Digital Economy Papers, No. 129, OECD publishing.

PONZELLINI A.M. (ed.), 2006, Quando si lavora con le tecnologie - Donne e uomini nelle professioni dell'ICT, Roma, Edizioni Lavoro.

RAMIOUL M., B. DE VROOM, 2009, Global value chain restructuring and the use of knowledge and skills, WORKS report. HIVA (KU Leuven) / Unibook. 
Cycles de vie et carrières dans les métiers des TIC :

une perspective de genre

SøRENSEN K., STEWART J., 2002, Digital Divides and Inclusion Measures - $A$ Review of Literature and Statistical Trends on Gender and ICT, Trondheim/ Edinburgh, NTNU.

TREMBLAY D.G., 2003, « Nouvelles carrières nomades et défis du marché du travail : une étude dans le secteur multimédia ", dans Revue de Carriérologie 9(2), Montréal, pp. 255-280.

VALCOURT M., P.S. TOLBERT, 2003, « Gender, family and career in the era of boundarylessness : determinants and effects of intra- and inter-organisational mobility », in International Journal of Human Resource Management 14(5), pp. 768-787.

VALENDUC G., P. VENDRAMIN, C. GUFFENS (FTU), A. PONZELLINI,, A. LEBANO (FRPS), L. D'OUVILLE, I. COLLET (ANACT), I. WAGNER, A. BIRBAUMER, M. TOLLAR (TUW), J. WEBSTER (RCWE), 2004a, Widening Women's Work in Information and Communication Technology, Final Report WWW-ICT (IST-2001-34520), Brussels, European Commission, July 2004.

VALENDUC G., P. VENDRAMIN, M. PEDACI, M. PIERSANTA, 2009, Changing careers and trajectories - How individuals cope with organizational change and restructuring. WORKS report. HIVA (KU Leuven) / Unibook.

VALENDUC G., P. VENDRAMIN, B.J. KRINGS, L. NIERLING, 2008, How restructuring is changing occupations - Case study evidence from knowledge-intensive, manufacturing and service occupations. WORKS report. HIVA (KU Leuven) / Unibook.

VALENDUC G., P. VENDRAMIN, C. GUFFENS, 2004b, « La place des femmes dans les métiers des technologies de l'information et de la communication ", dans Wallonie, (CERSW), n80, Liège, septembre 2004, pp. 40-49.

VALGAEREN E., 2007, Loopbanen van mannen en vrouwen in de ICT-sector, Doctoraat, Universiteit Antwerpen.

VENDRAMIN P., 2007, "Les métiers des TIC : un nomadisme coopératif ", dans De Saint-Laurent A.F. et J.L. Metzger (eds), Où va le travail à l'ère du numérique?, Presses de l'École des Mines, Paris, pp. 89-105.

VENDRAMIN P., 2004, Parcours professionnels dans les métiers des TIC, Namur, Rapport pour le Fonds social européen et la Région wallonne.

VENDRAMIN P., 2005, «TIC : écarts entre les hommes et les femmes en Europe ", dans les Actes du colloque Les femmes face aux sciences et technologies de l'information et de la communication, Paris, 5/12/2005. 
Gérard VALENDUC

WEBSTER J., 2003, "The Value of Men and Women : Gendering Knowledge and Skills in the Information Society », in Bechmann G., B.J. Krings, M. Rader (eds), Across the Divide: Work, Organization and Social Exclusion in the European Information Society, Berlin, Sigma Verlag, pp. 101-116. 